radiation force from a counterpropagating laser beam. The atoms are cooled further by a configuration of three orthogonal standing waves (Fig. 2) known as optical molasses, owing to the way the light exerts a damping force on the atoms so that they move like particles stuck in a viscous fluid. To build up a high density of atoms, a quadrupole magnetic field from a pair of coils with opposed currents was used in conjunction with the molasses laser beams to form a magneto-optical trap. The atoms are confined by an imbalance in radiation forces resulting from the Zeeman effect on the atomic energy levels (not by a direct magnetic force). Once a large number of cold atoms has accumulated at the centre of the trap, the current is turned off and the atoms are launched upwards using the light forces to form an atomic fountain; they travel for 30 $\mathrm{cm}$ before turning round and falling back down under gravity.

On their upward journey the atoms pass through a laser beam, which optically pumps them all into a single hyperfine level of the ground state before they enter the pair of counterpropagating beams that perform the velocity selection. The resulting velocity distribution is measured by passing the atoms through a small slit in a liquid-nitrogencooled shield into a region in which the atoms in the upper hyperfine level can be detected by counting the ions from laserphotoionization. The transverse width of the atom beam was that of the slit - there was no observable spreading. The upper limit for the transverse velocity was thus $1 \mathrm{~mm} \mathrm{~s}^{-1}$. The value of $0.27 \mathrm{~mm} \mathrm{~s}^{-1}$ quoted by Chu and colleagues was inferred from the measured linewidth of the Raman transition - it is difficult to measure velocities this small. In fact the authors showed that selection and detection of atoms across a range of initial velocities determines the initial distribution more accurately than the usual time-of-flight methods.

Kasevich and Chu have devised, in work yet to be published, an atom interferometer, analogous to an optical interferometer, that relies on the wave-like properties of atoms. The de Broglie wavelength of the atoms is 54 $\mu \mathrm{m}$, projected along the laser beams. Consider atoms which start in the lower level and with momentum $p$ along the laser beams; we denote these by $|1, p\rangle$. A first Raman pulse creates a superposition of the initial state $|1, p\rangle$ and $12, p+2 h / \lambda>$, in which the atom is in level 2 with additional momentum equal to twice the photon momentum. These wavepackets will move apart and become separated by as much as $2.4 \mathrm{~mm}$ in this initial experiment. A second Raman pulse, twice as long as the first, swaps over the states of the two packets so that they move back together. A third Raman pulse, with the same duration as the first, recombines the amplitudes - the final atomic state is a mixture of the two levels that depends on the relative phase difference of the two paths (and the laser fields). Kasevich and Chu based their matter-wave interferometer on this principle, but with the Raman beams vertical, to determine the gravitational acceleration of sodium atoms to a few parts per million.

Matter-wave interferometers have been made before using electrons and neutrons. But experiments with atoms are only now coming to fruition. Two groups (O. Carnal and J. Mlynek Phys. Rev. Lett. 66, 2689-2692; and D. W. Keith et al. 2693-2696; 1991) have just reported sucPARTICLE ACCELERATORS

\section{Focusing for free}

\section{Roger Evans}

WHEN most people think about focusing, whether for light waves or for charged particles, the mental picture is of carefully designed and exquisitely manufactured components of the closest possible tolerances. It comes as something of a surprise to find, as H. Nakanishi et al. have (Phys. Rev. Lett. 66, 1870-1873; 1991), that nature provides a near ideal focusing system for charged particles in nothing more than a puff of ionized gas.

The possible advantages of variants of this 'plasma lens' concept have been widely discussed by those operating particle accelerators in their quest for ever-higher-energy machines. Future multi-teraelectronvolt accelerators will have to focus their colliding beams down to almost atomic dimensions if interesting collisions are to occur at a sufficient rate to satisfy the experimenters.

Conventional quadrupole magnets focus beams in only one of the two transverse planes. The ability to focus charged particles in both planes simultaneously requires there to be a charge density or current in the focusing region. Controlling the uniformity of the necessary plasma has usually been thought to be insurmountable given the propensity of plasmas to collapse via a wide range of instabilities. But the new experiments by Nakanishi et al. show that the charge distribution required to focus the beam can be produced by the beam itself and is in this sense 'free', only a background plasma has to be provided. The mechanism is straightforward and depends on the fact that the particles in a highly relativistic beam in a vacuum experience competing focusing and defocusing forces: the electrostatic repulsion between the charged constituents is defocusing, but the magnetic attraction arising from their 'parallel currents' cancels out the repulsion to a factor depending on how close to the speed of light the beam travels.

When the beam passes through a background plasma, the plasma electrons will move in or out in an attempt to cancel the space charge, leaving the attractive electromagnetic force to pinch the beam particles. If the beam density is uniform the attractive force increases linearly with beam radius so that it is a near ideal focusing lens. Experi- cess in making atom interferometers using fine slits and diffraction gratings, respectively, to observe the interference of atomic wavepackets. Interferometers like these and that at Stanford based on laser-cooled atoms could be used in new tests of the remarkable subtleties of quantum mechanics.

Christopher Foot and Andrew Steane are in the Clarendon Laboratory, University of Oxford, Parks Road, Oxford OX1 3PU, UK. ments with 18-megaelectronvolt electrons at the University of Tokyo have confirmed this effect and shown that the quality of focusing is even better than a perfect lens. This paradoxical result is tentatively ascribed to a coupling of the transverse and longitudinal motions so that the all important 'phasespace volume' (a dynamic notion) is conserved.

Unfortunately, just as in economics, there are no 'free lunches'. This free focusing has some drawbacks. In its passive form, as described above, the strength of the focusing action increases as the beam passes through the plasma, the tail is focused much more strongly than the head which is not very practical for a colliding-beam system. The focusing is symmetrical for positive and negative particles only if the beam density is much less than the plasma density, in which case one might worry about scattering from the plasma particles. A practical disadvantage is that the focusing lens appears to move as the input beam moves which makes the alignment tolerances much more critical for two beams to collide at the same point.

Novel ideas such as this plasma lens or plasma-based accelerators have been part of the imaginative schemes put forward for particle accelerators to advance to the next generation. Although the new ideas always have some highly attractive features it is surprising just how resilient and ingenious are the designers of conventional accelerators and so far all serious designs have been based on radiofrequency acceleration and magnets for bending and focusing. The plasma lens is unlikely to be used as the sole focusing element but its cheapness and simplicity make it the ideal way to enhance existing systems. A passive plasma lens close to the focus of two colliding beams may well provide a useful increase in the luminosity at the intersection for very little cost. But its use in circular machines is sadly limited: as the action is not reversible, the focused beam cannot be recollimated; and the accumulation of scattering from plasma particles will eventually destroy the beam quality. $\square$

Roger Evans is at the Rutherford Appleton Laboratory, Didcot OX11 OQX, UK. 